\title{
Tuning and Sensitivity Improvement of Bi-Metallic Structure-Based Surface Plasmon Resonance Biosensor with 2-D $\varepsilon$-Tin Selenide Nanosheets
}

\author{
Natarajan Sathya ${ }^{1} \cdot$ Bhishma Karki ${ }^{2}$ Kantilal Pitambar Rane ${ }^{3} \cdot$ Ankit Jha $^{4} \cdot$ Amrindra Pal $^{4}$ (D) \\ Received: 20 June 2021 / Accepted: 18 November 2021 / Published online: 18 January 2022 \\ (c) The Author(s), under exclusive licence to Springer Science+Business Media, LLC, part of Springer Nature 2021
}

\begin{abstract}
This manuscript aims to analyze the effect of tin selenide ( $\mathrm{SnSe}$ ) on the sensing application of SPR biosensors. Tin selenide is the 2-dimensional transition metal dichalcogenide material. The proposed multilayer structure has a BK7 prism, a bimetallic layer of $\mathrm{Au}$, tin selenide, and a graphene layer. Tin selenide is used to improve the performance parameters of the biosensor. The $\varepsilon-\mathrm{SnSe}$ nanosheet is placed in between two layers of gold ( $\mathrm{Au})$ in the Kretschmann configuration. The proposed configuration has a maximum sensitivity of $214 \mathrm{deg} / \mathrm{RIU}, 93.81 \%$ higher than the conventional sensor. The performance parameters like full width half maximum, detection accuracy, and quality factor have been analyzed. The $\varepsilon-$ SnSe material is an air-stable 2-D. The proposed sensor is suitable for the analysis of chemical, medical, and biological analytes.
\end{abstract}

Keywords Surface plasmon resonance $\cdot$ Sensitivity $\cdot \varepsilon$-Tin selenide nanosheets $\cdot$ Biosensor

\section{Introduction}

The tremendous development of surface plasmon resonance (SPR) technology has been reported for biomedical applications in the last few decades. The biosensor can detect cancer cells, DNA hybridization, antibody characterization, protein conformation, and the recent detection of the CORONA virus. In the biosensing field, to detect, analyze, and characterize the biomolecule, chemical, environment, and food [1-5], these biosensors are highly sensitive and facilitate real-time analysis of the contaminated analyte [6-8]. The SPR biosensor works on the phenomenon of attenuated total reflection (ATR). As the refractive index of the sensing layer varies $[9,10]$, a sharp dip in the resonance curve is obtained due to the adsorption of the incident light in the sensing medium $[11,12]$. The Kretschmann

Amrindra Pal

1 Engineering Department, Scientific Society Group, Tamilnadu, India

2 Department of Physics, Tri-Chandra Multiple Campus, Tribhuvan University, Kathmandu 44600, Nepal

3 KCE COEM Jalgaon, Jalgaon, India

4 Department of EECE, DIT University, Dehradun, Uttrakhand 248009, India configuration is the most acceptable configuration for designing the biosensor $[13,14]$. This configuration has a prism, a thin layer of metal, and dielectric material. The layer of metal is used to generate the surface plasmons. The metal has poor adsorbability, so few layers of 2-dimensional material are associated with enhancing the biosensors' performance [15-19]. When an incident light beam propagates through two media interfaces with the different refractive indexes at a particular angle and different dielectric constants, the surface plasmon gets excited [20]. These surface plasmons are electromagnetic waves traveling along the surface of the interface.

Single-layer SnSe also possesses fantastic electrical and optical properties and generates high interest as a 2D material beyond the predecessor members, displaying the same structure as graphene and phosphorene [21-23]. As a classical p-type IV-VI semiconductor, SnSe has a narrow gap (1.30 eV direct and $0.90 \mathrm{eV}$ indirect), lower toxicity, and higher chemical stability [24]. That SnSe has a layered crystal structure similar to other IV-VII binary semiconductors, such as $\mathrm{SnSe}, \mathrm{GeS}$, and GeSe. In experiments, the assumption that unilaminar crystal $\mathrm{SnSe}$ would display different thermoelectric properties on different axes was authenticated by Zhao et al. [18], who manufactured hole-doped single-crystal SnSe [20]. A lot of SnSe allotropes have been investigated by density functional theory [18]. Monolayer 
SnSe has outstanding thermoelectric properties and is also an eminent optoelectronic material. Still, there are few systematic studies on the optic and electric characteristics of $\mathrm{SnSe}$ allotropes. A metal-like Gold ( $\mathrm{Au}$ ) is not susceptible to oxidation and does not react with most chemicals, and hence it is often used as the metal film in sensors. At the metal-dielectric interface, the intensity of the incident light beam reaches a maximum and decays exponentially into media [25]. Since the metal and wave interaction is lossy, the EM field of a surface plasmon is concentrated in a vast majority in the dielectric medium close to the metal surface. The SPR phenomenon results in a graded reduction in the intensity of the reflected light. Surface plasmon resonance (SPR) sensors have generated considerable interest since they allow real-time detection of biomolecular interactions, rapid, level free, and valuable diagnostic tool for diseases having virus size of the order of nanometer or less [26].

In particular, graphene has attractive properties such as tunable electrical and optical characteristics [27], ring-type carbon structure and high surface-to-volume ratio. Thus, the adsorbates can easily interact with this structure, increasing the adsorption ability of the biosensors [28, 29].

Enhancing the sensor's sensitivity is an emerging issue studied at different places; different methods and strategies have been proposed. The metals are used as plasmonics materials to generate the surface plasmons. $\mathrm{Ag}$ and $\mathrm{Au}$ are the most commonly used metals for the SPR sensor [30]. SPR technique help in estimating the dimension of DNA [31], RNA, or blood cells which cannot be measured directly due to its sizes being less than a nanometer. Therefore, the indirect measurement technique, which was evolved in 1968 by Kretschmann, opened a new avenue where the refractive index variation interacts with incident light photon and the reflected ray, thus quantifying the size or the nature of the virus under investigation. The binding of the legend differs; therefore, SPR has successfully detected the diverse effect and resulting refractive index change [32,33].

The manuscript is sectioned as follows, the "Theoretical Model and Design Consideration" section proposed theoretical model and design consideration for the proposed biosensor. The "Results and Discussions" section consists of the results and discussion, and finally, the "Conclusions" section concludes the proposed work.

\section{Theoretical Model and Design Consideration}

We consider a modified biosensor configuration, as shown in Fig. 1. Each layer of the materials is assumed to be stacked along the $z$-axis. BK7 is chosen as a coupling prism in our structure, which is covered with one layer of gold-1, $\varepsilon-$ SnSe layer is close to gold-1 layer, and the other side of $\varepsilon-$ SnSe layer also connects with another gold-2 layer. Graphene layer is used as a biomolecular recognition element, which is close to the gold-2 layer. Besides, the thickness of the two layers of gold is not the same. We set the thickness of two layers of gold as $W_{1}=40 \mathrm{~nm}$ and $W_{3}=20 \mathrm{~nm}$, respectively. The thickness of $\varepsilon-\mathrm{SnSe}$ is $W_{2}=\mathrm{SnSe} \times 1.5$ $\mathrm{nm}$, where $\mathrm{SnSe}$ is the layer number of $\varepsilon-\mathrm{SnSe}$ sheets. Graphene is connected with the sensing layer for increasing the ability of macromolecular adsorption of the biosensor, and the thickness of graphene is $W_{4}=G \times 0.34 \mathrm{~nm}$, where $G$ is the layer number of graphene sheets. A TM-polarized light $633 \mathrm{~nm}$ is assumed to be an incident from one side of the prism and receives reflected light from the other. The received data can be used to calculate the reflectivity and sensitivity of the structure.
Fig. 1 Heterostructure design of the proposed biosensor

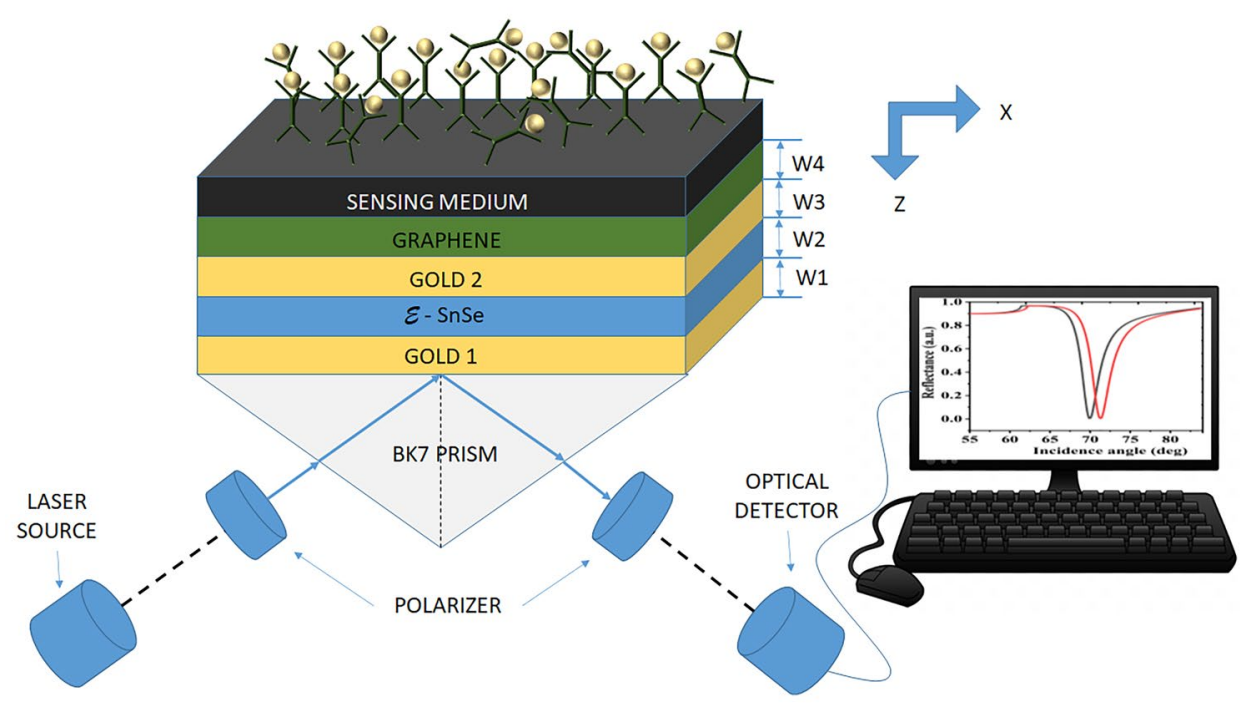



[34]

The refractive index $\left(n_{\text {Prism }}\right)$ of BK7 layer is expressed as where $n_{0}$ is the RI of the sensing layer, $\epsilon_{s}$ and $\epsilon_{m}$ are the dielectric constant for the sensing layer and analyte/metal,

$n_{\text {Prism }}=\left(\frac{1.03961212 \lambda^{2}}{\lambda^{2}-0.00600069867}+\frac{0.231792344 \lambda^{2}}{\lambda^{2}-0.0200179144}+\frac{1.0104694 \lambda^{2}}{\lambda^{2}-103.560653}+1\right)^{\frac{1}{2}}$

where $\lambda$ is the wavelength of the applied optical signal.

The RI of the metal can be computed using the Drude model [35] by the formula:

$n_{1,3}(\lambda)=\sqrt{1-\frac{\lambda^{2} \lambda_{c}}{\lambda_{p}^{2}\left(\lambda_{c}-i \lambda\right)}}$, where $\lambda_{p}$ and $\lambda_{c}$ are the wavelengths of gold for plasma and collision and are taken as $1.6826 * 10^{-7} \mathrm{~m}$ and $8.9342 * 10^{-5} \mathrm{~m}$, respectively [36].

The refractive index of $\varepsilon-\mathrm{SnSe}$ is considered as $4.4+3.53 i[25]$ in the visible region. In the proposed multilayered structure, reflectivity is calculated by formula as follows:

$r_{p m_{1} d_{1} m_{2} d_{2}}=\frac{r_{p m_{1}}+r_{m_{1} d_{1} m_{2} d_{2}} e^{2 i K m_{1} x d m_{1}}}{1+r_{p m_{1}} r_{m_{1} d_{1} m_{2} d_{2}} e^{2 i K m_{1} x d m_{1}}}$

where

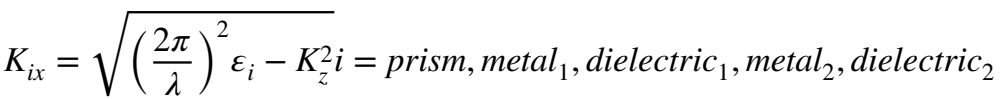

$\theta_{S P R}$ is resonance angle, $K_{z}$ is the propagation constant in the z-direction of the incident light, and $K_{s p}$ is propagation constant. The RI of the graphene film is given as $3+1.1487 i$ [22]. The RI of sensing film is taken as $n_{s}=1.373$. The sensitivity of the biosensor is represented as $S=\delta \theta / \delta n$, where $\delta n$ is the change in the RI. The detection accuracy (DA) of the biosensor can be expressed as $D A=1 / F W H M$, where the full-width half maximum (FWHM) is the width of the spectra of the SPR curve at that point, the reflectivity is $50 \%$. The FWHM should be small. The high value of the sensitivity and DA is desirable [37]. The quality factor can be expressed as $Q=S . D A=S / F W H M$, directly depends on the FWHM and sensitivity. Table 1 shows the optimized parameters selected for the theoretically examined biosensor. $r_{\text {prismmetal }}=\frac{\left(\varepsilon_{\text {metal }} K_{\text {prismx }}-\varepsilon_{\text {prism }} K_{\text {metal }}\right)}{\left(\varepsilon_{\text {metal }} K_{\text {prismx }}+\varepsilon_{\text {prism }} K_{\text {metalx }}\right)}$

$r_{\text {metaldielectric }}=\frac{\left(\varepsilon_{\text {dielectric }} K_{\text {metalx }}-\varepsilon_{\text {metal }} K_{\text {dielectricx }}\right)}{\left(\varepsilon_{\text {dielectric }} K_{\text {metalx }}+\varepsilon_{\text {metal }} K_{\text {dielectricx }}\right)}$

The condition for resonance is fulfilled using Eqs. 2 and 3. The equation computes the sharp downfall in the resonance curve:

$K_{z}=K_{s p}=\frac{2 \pi}{\lambda} n_{0} \operatorname{Sin} \theta_{S P R}=\operatorname{real}\left(\frac{2 \pi}{\lambda} \sqrt{\frac{\epsilon_{m} \epsilon_{s}}{\epsilon_{m}+\epsilon_{s}}}\right)$

\section{Results and Discussions}

Emphasis has been made to improve the sensitivity and performance of biosensor low refractive index prism is used. $\mathrm{BK} 7$ prism is most suitable because it has a low refractive index. If the prism has a high refractive index, it provides a sharp dip in the resonance curve compared to the low RI prism. Low refractive prism provides better values of the FWHM, angle of resonance, the shift in the resonance curve, and sensitivity than the high refractive index's prism. To get the high sensitivity and low FWHM, the flat end of the prism is polished with a 40-nm thick gold layer. A layer of the $\mathrm{SnSe}$ is deposited on the top of the Au layer. Another $\mathrm{Au}$ layer of $20 \mathrm{~nm}$ is deposited over the $\mathrm{SnSe}$ layer to enhance the sensor's sensitivity. A single metal layer cannot increase
Table 1 Optimized parameters for the proposed biosensor

\begin{tabular}{lllll}
\hline Film of the materials & Used material & \multicolumn{2}{l}{ RI of the material at 633 nm } & Thickness (nm) \\
\cline { 3 - 4 } & & Real part $(\boldsymbol{n})$ & $\begin{array}{l}\text { Imaginary } \\
\text { part }(\boldsymbol{k})\end{array}$ & \\
\hline Layer I & Prism BK7 & 1.5151 & - & - \\
Layer II & Au (Metal) & 0.19572 & 3.2561 & $w_{1}=40$ \\
Layer III & $\varepsilon-$ Tin selenide & 4.4 & 3.53 & $w_{2}=\mathrm{SnSe} * 1.5$ \\
Layer IV & Au (metal) & 0.18377 & 3.4313 & $w_{3}=20$ \\
Layer V & Graphene & 3 & 1.1487 & $w_{4}=\mathrm{G} * 0.34$ \\
\hline
\end{tabular}


sensitivity too much, so a bimetallic layer is used. In the end, the graphene layer is spread over the gold-2 layer.

Figure 2 shows the reflectance plot of the biosensor. Figure $2 \mathrm{a}$ shows the design of a conventional sensor having two layers of $\mathrm{Au}$ and a sensing layer, i.e., $\mathrm{SnSe}=G=0$. A sharp dip in the resonance curve at a specific angle is obtained because SRs are excited. It clearly states that the absorption of the incident light occurs due to the SPs' generation. The excursion at the dip is $\delta \theta=0.97^{\circ}$, and sensitivity $194 \mathrm{deg} /$ RIU is obtained. A single layer of the $\mathrm{SnSe}$ is considered, and the graphene layer is missing, i.e., $\mathrm{SnSe}=1, G=0$, the sensitivity is enhanced due to the absorbance of the incident light. The change in the resonance angle $\delta \theta=0.98^{\circ}$ is obtained (shown in Fig. 2b), and sensitivity of $196 \mathrm{deg} / \mathrm{RIU}$ has been achieved. These values are better than the values obtained from the conventional biosensor. This improvement
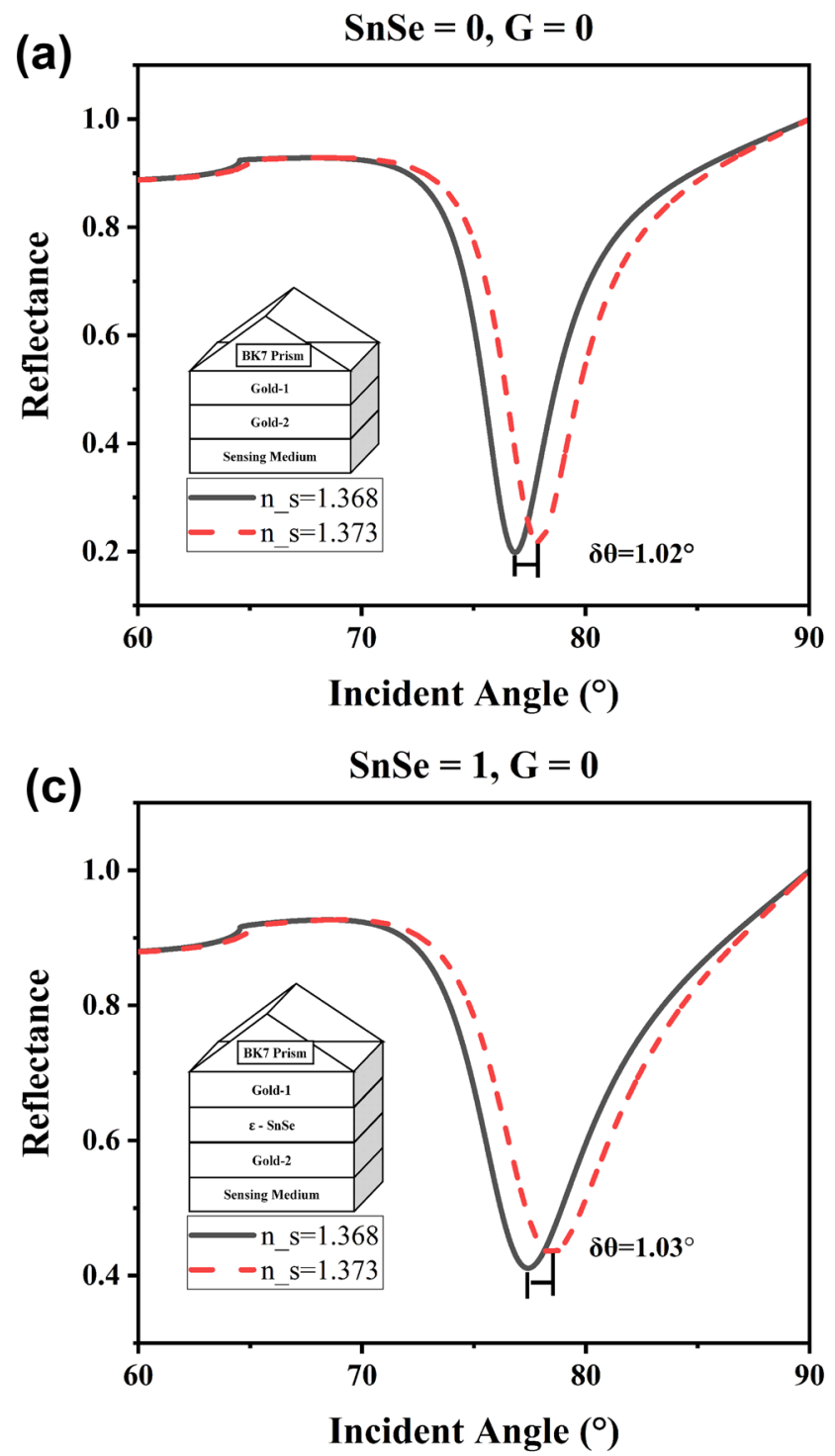

in the performance is attributed to the narrow bandgap of the SnSe material and better absorption efficiency.

A single graphene layer is present, and the SnSe layer is missing in the structure, i.e., $\mathrm{SnSe}=0, G=1$. Now, the impact of the graphene is analyzed, and it is found that offset resonance dip and sensitivity both are improvised, and values of $\delta \theta=0.99^{\circ}$ and $198 \mathrm{deg} / \mathrm{RIU}$ are claimed respectively (shown in Fig. 2c). The enhanced sensitivity and the increase in resonance curve offset dip in this structure are attributed to the higher adsorption rates due to the large surface area and rich $\pi$ conjugation structure offered by graphene, making it the appropriate choice for dielectric top layer SPR sensing applications $[27,38]$.

Another scenario was evaluated for the impact by introducing a single layer of $\mathrm{SnSe}$ and a monolayer of graphene in the biosensor ( $\mathrm{SnSe}=1, G=1$ ). This results in significant
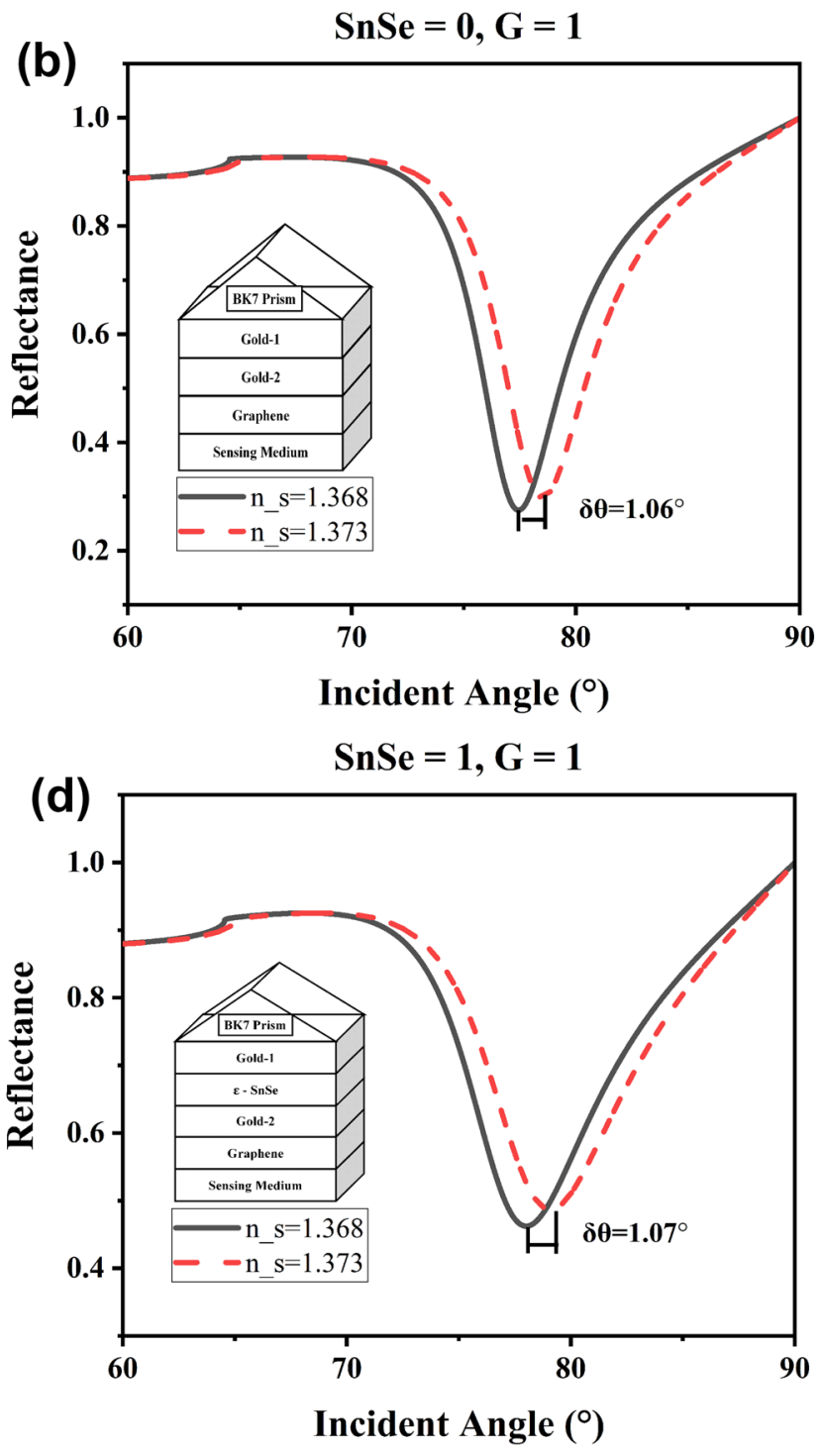

Fig. 2 Reflectance vs. incident angle (deg) at the same RI change with $\varepsilon-\mathrm{SnSe}$ and graphene (a) $\mathrm{SnSe}=0, G=0,(\mathbf{b}) \mathrm{SnSe}=0, G=1,(\mathbf{c})$ $\mathrm{SnSe}=1, G=0,(\mathbf{d}) \mathrm{SnSe}=1, G=1$ 
shifts and a dip in the resonance curve. The resonance angle was obtained as $\delta \theta=1.02^{\circ}$, whereas the sensitivity was $214 \mathrm{deg} / \mathrm{RIU}$ as depicted in Fig. 2d. This comparative study reveals that the resonance angle offset increases with adding a single layer of graphene, a single layer of $\mathrm{SnSe}$, compared to the conventional sensor. The best results were obtained by adding a single layer of graphene and a single layer of $\mathrm{SnSe}$. Table 2 consists of performance parameters that are directly obtained from the theoretical study of the proposed work. Based on our knowledge, few parameters are better reported than the previously existing literature.

By comparing the relevant data, we can find that the resonance angle offset of the biosensor in our structure is larger than the traditional SPR structure due to the addition of graphene and the $\varepsilon-$ SnSe layer. The traditional SPR structure contains only a prism and gold layer. Hence, we can conclude that the sensitivity of biosensors in our structure has significantly improved compared with the traditional design by adding graphene and $\varepsilon-$ SnSe layer.

From the analyses made above, it is clear that the sensing layer's refractive index can control the biosensor's sensitivity. The enhancement of the sensitivity of the biosensor with graphene and $\varepsilon-$ SnSe layers is more than the single layer of these materials. The sensitivity of the sensor also depends on the RI of the sensing medium. Here, we select the sensing layer refractive index range from 1.368 to 1.373 . It can be seen that as the refractive index of the sensing layer increases from 1.368 to 1.373 , the sensitivity increases significantly (Fig. 3).

Figure $4 \mathrm{a}$ shows the impact of $\varepsilon-\mathrm{SnSe}$ layers on the sensitivity of the biosensor. It is evident that with the introduction of several $\varepsilon-$ SnSe sheets, reflectance increases. Thus, the reflectance dip transfers to a larger incident angle and broadening of the SPR curve. Similarly, the effect of the change of the graphene layer on the sensitivity is given in Fig. 4b. A similarity in results with $\varepsilon-\mathrm{SnSe}$ is observed. The reflectance dip transfers to a larger incident angle, and the broadening of the SPR curve takes place as the number of graphene layers is increased from 1 through 4 . Thus, for analysis purposes, the values of $S n S e$ and $G$ are confined to 0 and 1. For the values SnSeand $G=2$ (and higher), it was observed that the shift

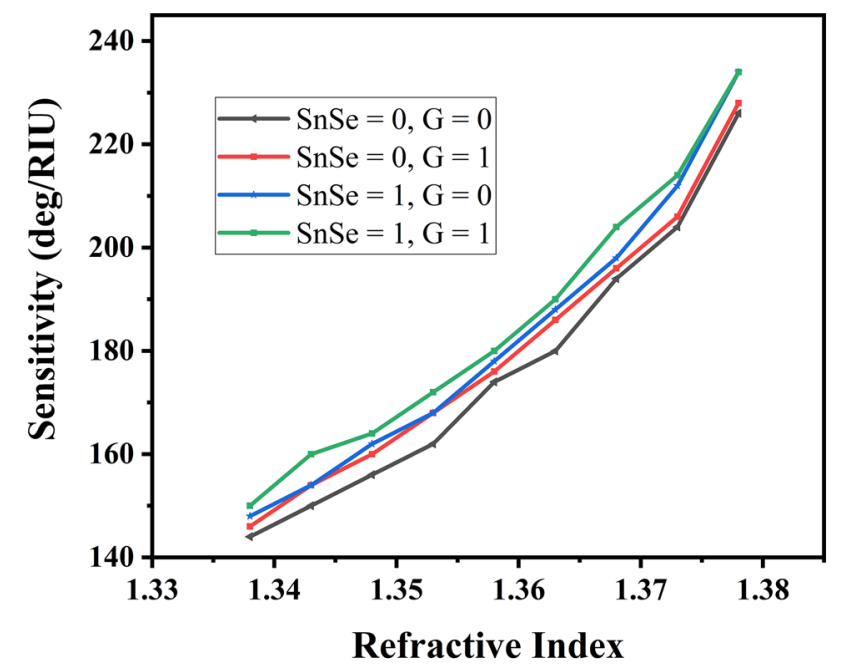

Fig. 3 A plot of sensitivity vs. sensing layers refractive index

in reflectance curve for various values of refractive indices of sensing layer is overlapping, or one of the reflectances was over 0.5 makes. It is challenging to measure accurate resonance angle in the dip for these values and lower sensitivity. Upon further analysis of the results as reported in Fig. 4a, b, it can be observed that the resonant angle offset is more affected by the increase in the number of $\mathrm{SnSe}$ sheets than the corresponding increase in the number of graphene layers.

Figure 5 shows the graphical representation of the performance parameters of the investigated sensor. Figure 5a shows the variation in FWHM and minimum reflectance to sensitivity. Figure $5 \mathrm{~b}$ shows the detection accuracy and quality factor.

Table 3 comprises the performances of the biosensor for the maximum change in the RI is 0.005 , the thickness of the Au layers is chosen $40 \mathrm{~nm}$ and $20 \mathrm{~nm}$, the thickness of the $\varepsilon-\mathrm{SnSe}$ is $1.5 \mathrm{~nm}$, and thickness of the graphene 0.34 . Table 4 reports the comparative analysis of the earlier published work. Table 4 reflects that all the referenced work is published, and it can consider that all are standard. The proposed work here shows the best performance parameters as well as high sensitivity.

Table 2 Final values of the different parameters for the sensor

\begin{tabular}{|c|c|c|c|c|c|c|c|}
\hline Structures (prism BK7) & $\begin{array}{l}\text { Resonance } \\
\text { angle }\left(\boldsymbol{\theta}_{\text {SPR }}\right)\end{array}$ & $\delta \boldsymbol{\theta}$ & FWHM & Sensitivity & Quality factor & DA & $\boldsymbol{R}_{\min }$ \\
\hline 1. Bimetallic layer $(\mathrm{Au})$ & 76.86 & 0.97 & 4.434 & 194 & 46.01 & 0.23 & 0.22 \\
\hline 2. Bimetallic layer $+\varepsilon-\mathbf{S n S e}$ & 77.42 & 0.98 & 7.039 & 196 & 30.12 & 0.18 & 030 \\
\hline 3. Bimetallic layer + graphene & 77.44 & 0.99 & 5.41 & 206 & 38.08 & 0.14 & 0.43 \\
\hline 4. Bimetallic layer + graphene $+\varepsilon-\mathbf{S n S e}$ & 78.02 & 1.02 & 7.947 & 214 & 26.93 & 0.13 & 0.49 \\
\hline
\end{tabular}




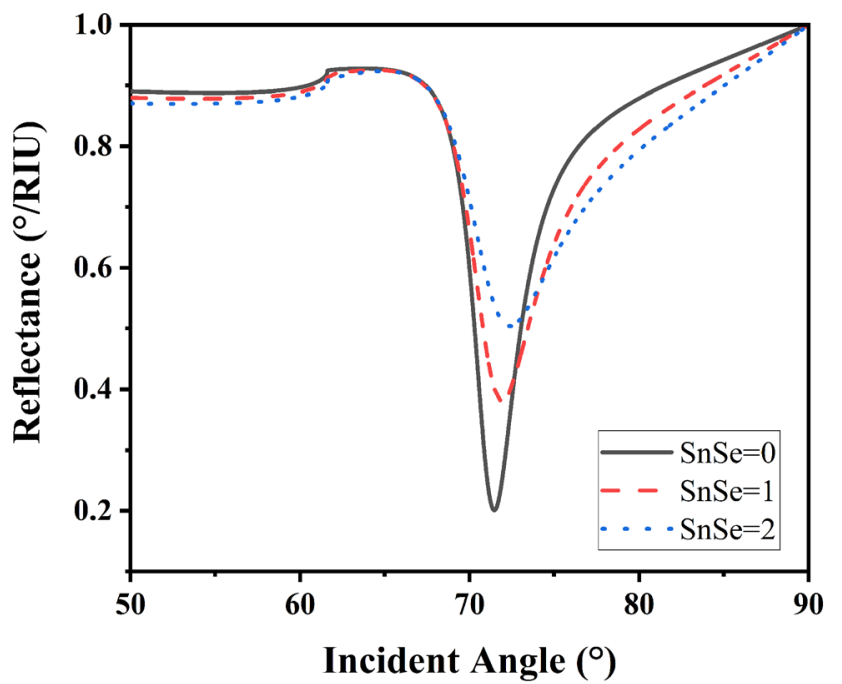

(a)

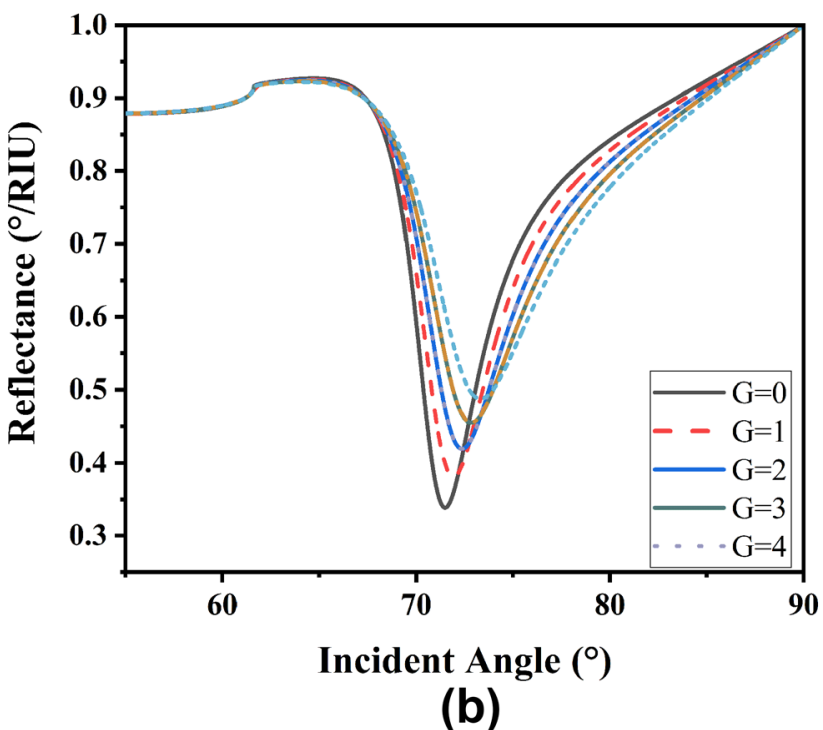

(b)

Fig. 4 (a, b) Change in the reflectance w.r.t incident angle (a) variation in the number of tin selenide layers at one graphene layer and (b) variation in the number of graphene layers at one tin selenide

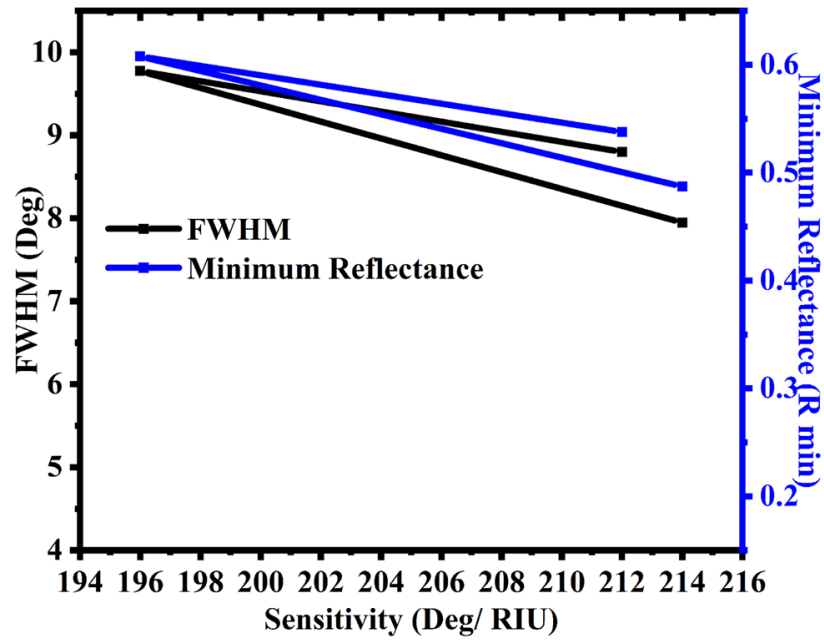

(a)

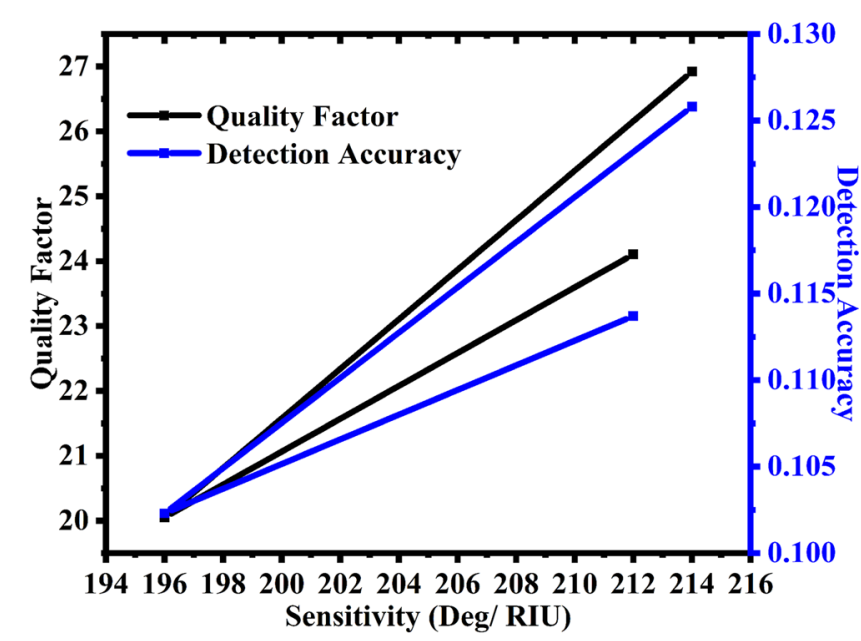

(b)

Fig. 5 Graphical representation comparing (a) the sensitivity with FWHM and minimum reflectance of $\varepsilon$ - SnSe layers and graphene layer, (b) the sensitivity with quality factor and detection accuracy of $\varepsilon-$ SnSe layers and the graphene layer

Table 3 Depicts the analytical values at which the quality factor obtained is maximum

\begin{tabular}{llllll}
\hline Layers & FWHM & Detection accuracy & Sensitivity & Quality factor & $\begin{array}{l}\text { Minimum } \\
\text { reflectance }\end{array}$ \\
\hline $\mathrm{SnSe}=1 \mathrm{G}=1$ & 7.947 & 0.1258 & 214 & 26.9 & 0.487 \\
$\mathrm{SnSe}=2 \mathrm{G}=1$ & 9.774 & 0.1023 & 196 & 20.1 & 0.6077 \\
$\mathrm{SnSe}=1 \mathrm{G}=2$ & 8.798 & 0.1137 & 212 & 24.1 & 0.5376 \\
\hline
\end{tabular}


Table 4 Comparative analysis with the earlier reported work

\begin{tabular}{llll}
\hline Layers & Wavelength $\lambda$ & $\begin{array}{l}\text { Sensitivity } \\
(\mathrm{deg} / \text { RIU })\end{array}$ & Reference \\
\hline Chromium, gold, SnSe nanosheet, sensing layer & $633 \mathrm{~nm}$ & 160 & {$[39]$} \\
Silver, SnSe, sensing layer & $633 \mathrm{~nm}$ & 154 & {$[25]$} \\
Gold, SnSe, gold, graphene, sensing layer & $633 \mathrm{~nm}$ & 214 & Proposed work \\
\hline
\end{tabular}

\section{Conclusions}

The proposed biosensor is numerically simulated, and performance parameters are analyzed. The proposed sensor is a modified Kretschmann configuration-based SPR sensor with a bimetallic layer of gold, $\varepsilon-\mathrm{SnSe}$, and graphene nanosheetbased modified. The study is carried out at $633 \mathrm{~nm}$ operating wavelength, and the highest sensitivity, $214 \mathrm{deg} / \mathrm{RIU}$, is obtained for the monolayers of the $\varepsilon-$ SnSe allotrope and graphene. The thickness of the gold layers is 40 and $20 \mathrm{~nm}$, respectively is taken in this work. The refractive index of the medium is taken as 1.368. The proposed sensor is useful for the study of the chemical, environment, biomolecule, and analytes.

Author Contributions NS formulated the problem statement wherein giving the theoretical background for SPR biosensor. He also helped in drafting the manuscript. BK formulated the problem statement and improvised the quality of the figure and graph. KPR provided the theoretical background to biosensing and the importance of optical biosensing. He also helped in reviewing the manuscript. AJ provided statistical analysis for the results. He provided the theoretical background to SPR biosensors. AP provided statistical analysis for the results. He provided the theoretical background to SPR biosensors. He also helped in reviewing and formatting the manuscript.

\section{Declarations}

Ethics Approval Not applicable. The work presented in this manuscript is mathematical modeling only for the proposed biosensor. No experiment was performed on the human body and living organism/animal. So, ethical approval from an ethical committee is not required.

Consent to Participate All the authors are willing to participate in the work presented in this manuscript. On behalf of all authors, communicating author is authorized to give their consent.

Consent for Publication All the authors have given their consent to publish this work.

Conflict of Interest/Competing Interests The authors declare no competing interests.

\section{References}

1. Bellassai N, D'Agata R, Jungbluth V, Spoto G (2019) Surface plasmon resonance for biomarker detection: advances in noninvasive cancer diagnosis. Front Chem 7(August):1-16
2. Asif M et al (2020) The role of biosensors in coronavirus disease-2019 outbreak. Curr Opin Electrochem 23(January):174-184

3. Nguyen HH, Park J, Kang S, Kim M (2015) Surface plasmon resonance: a versatile technique for biosensor applications. Sensors 15(5): 10481-10510

4. Taha BA et al (2020) An analysis review of detection coronavirus biosensor application. Sensors 20(23):6764

5. Mauriz E (2020) Recent progress in plasmonic biosensing schemes for virus detection. Sensors (Switzerland) 20(17):4745

6. Ahmed A, Rushworth JV, Hirst NA, Millner PA (2014) Biosensors for whole-cell bacterial detection. Clin Microbiol Rev 27(3):631-646

7. Bhalla N, Jolly P, Formisano N, Estrela P (2016) Introduction to biosensors. Essays Biochem 60(1):1-8

8. Hossain MB, Tasnim T, Abdulrazak LF, Rana MM, Islam MR (2020) A numerical approach to design the Kretschmann configuration based refractive index graphene-MoS2 hybrid layers with $\mathrm{TiO} 2-\mathrm{SiO} 2$ nano for formalin detection. Photonic Sens 10(2):134-146

9. Akowuah EK, Gorman T, Haxha S (2009) Design and optimization of a novel surface plasmon resonance biosensor based on Otto configuration. Opt Express 17(26):23511

10. Li L et al (2017) Dual Kretschmann and Otto configuration fiber surface plasmon resonance biosensor. Opt Express 25(22):26950

11. Karki B, Pal A, Singh Y, Sharma S, Srivastava V (2021) Sensitivity enhancement of surface plasmon resonance sensor using $2 \mathrm{D}$ material barium titanate and black phosphorus over the bimetallic layer of $\mathrm{Au}, \mathrm{Ag}$, and $\mathrm{Cu}$. Opt Commun

12. Karki B, Sharma S, Pal A, Singh Y (2021) Sensitivity enhancement of surface plasmon resonance biosensor with 2-D Franckeite nanosheets. Plasmonics

13. Pal A et al (2020) Prism based surface plasmon resonance biosensor for biomedical applications, in ICOL-2019, Springer Proceedings in Physics 258. Springer Singapore, pp 1-4

14. Zeng $Y$ et al (2020) Phase interrogation SPR sensing based on white light polarized interference for wide dynamic detection range. Opt Express 28(3):3442

15. Singh Y, Raghuwanshi SK (2019) Electromagnetic wave sensors sensitivity enhancement of the surface plasmon resonance gas sensor with black phosphorus. IEEE Sensors Lett 3(12):1-4

16. Pal A, Jha A (2021) A theoretical analysis on sensitivity improvement of an SPR refractive index sensor with graphene and barium titanate nanosheets. Optik (Stuttg) 231:166378

17. Homola J (2003) Present and future of surface plasmon resonance biosensors. Anal Bioanal Chem 377(3):528-539

18. Zhao LD et al (2014) Ultralow thermal conductivity and high thermoelectric figure of merit in SnSe crystals. Nature 508(7496):373-377

19. Hu ZY, Li KY, Lu Y, Huang Y, Shao XH (2017) High thermoelectric performances of monolayer SnSe allotropes. Nanoscale 9(41): 16093-16100

20. Zhao LD et al (2016) Ultrahigh power factor and thermoelectric performance in hole-doped single-crystal SnSe. Science (80-.) 351(6269):141-144

21. Verma A, Prakash A, Tripathi R (2015) Performance analysis of graphene based surface plasmon resonance biosensors for 
detection of pseudomonas-like bacteria. Opt Quantum Electron 47(5):1197-1205

22. Sharma AK (2018) Blue phosphorene/MoS 2 heterostructure based SPR sensor with enhanced sensitivity. IEEE Photon Technol Lett

23. Meshginqalam B, Barvestani J (2018) Performance enhancement of SPR biosensor based on phosphorene and transition metal dichalcogenides for sensing DNA hybridization. IEEE Sens J 18(18):7537-7543

24. Wei PC et al (2016) The intrinsic thermal conductivity of SnSe. Nature 539(7627):E1-E2

25. Dai X, Liang Y, Zhao Y, Gan S, Jia Y, Xiang Y (2019) Sensitivity enhancement of a surface plasmon resonance with tin selenide (SnSe) allotropes. Sensors (Switzerland) 19(1):1-8

26. Amendola V, Pilot R, Frasconi M, Maragò OM, Iatì MA (2017) Surface plasmon resonance in gold nanoparticles: a review. J Phys Condens Matter 29(20)

27. Rathi S et al (2015) Tunable electrical and optical characteristics in monolayer graphene and few-layer MoS2 heterostructure devices. Nano Lett 15(8):5017-5024

28. Aksimsek S, Jussila H, Sun Z (2018) Graphene - MoS 2 metal hybrid structures for plasmonic biosensors. Opt Commun 428:233-236

29. Mudgal N, Singh PYJAG (2020) BaTiO 3 -graphene-affinity layer - based surface plasmon resonance (SPR) biosensor for pseudomonas bacterial detection. Plasmonics 15:1221-1229

30. Zhang N, Han C, Fu X, Xu YJ (2018) Function-oriented engineering of metal-based nanohybrids for photoredox catalysis: exerting plasmonic effect and beyond. Chem 4(8):1832-1861

31. Mathur D, Medintz IL (2019) The growing development of DNA nanostructures for potential healthcare-related applications. Adv Healthc Mater 8(9)
32. Ritzefeld M, Sewald N (2012) Real-time analysis of specific protein-DNA interactions with surface plasmon resonance. J Amino Acids 2012:1-19

33. Terao K et al (2012) Size-exclusion SPR sensor chip: application to detection of aggregation and disaggregation of biological particles. Analyst 137(9):2192-2198

34. Srivastava A, Prajapati YK (2020) Effect of sulfosalt and polymers on performance parameter of SPR biosensor. Opt Quantum Electron 52(10):1-14

35. Pang K, Dong W, Zhang B, Zhan S, Wang X (2016) A performanceenhanced bimetallic chip for the detection of cadmium ions with surface plasmon resonance. Plasmonics 11(4):1119-1128

36. Brahmachari K, Ray M (2013) Effect of prism material on design of surface plasmon resonance sensor by admittance loci method. Front Optoelectron 6(2):185-193

37. Gupta BD, Verma RK (2009) Surface plasmon resonance-based fiber optic sensors: principle, probe designs, and some applications. J Sensors 2009:1-12

38. Murata H, Saitoh N, Yoshizawa N, Suemasu T, Toko K (2017) High-quality multilayer graphene on an insulator formed by diffusion controlled Ni-induced layer exchange. Appl Phys Lett 111(24):3-7

39. Varasteanu P (2021) Optimizing the tin selenide (SnSe) allotrope/ gold-based surface plasmon resonance sensors for enhanced sensitivity. Plasmonics 16(2):341-347

Publisher's Note Springer Nature remains neutral with regard to jurisdictional claims in published maps and institutional affiliations. 